\title{
Time-Delay Multiplexing of Two Beams in a Terahertz Imaging Radar
}

\author{
Nuria Llombart, Member, IEEE, Ken B. Cooper, Member, IEEE, Robert J. Dengler, Member, IEEE, \\ Tomas Bryllert, Goutam Chattopadhyay, Senior Member, IEEE, and Peter H. Siegel, Fellow, IEEE
}

\begin{abstract}
We demonstrate a time-delay multiplexing technique that doubles the frame rate of a 660-690-GHz imaging radar with minimal additional instrument complexity. This is done by simultaneously projecting two offset, orthogonally polarized radar beams generated and detected by a common source and receiver. Beam splitting and polarization rotation is accomplished with a custom designed waveguide hybrid coupler and twist. A relative time lag of approximately $2 \mathrm{~ns}$ between the beams' waveforms is introduced using a quasi-optical delay line, followed by spatial recombination using a selectively reflective wire grid. This delay is much longer than the approximately 20 -ps time-of-flight resolution of the 30-GHz bandwidth radar, permitting the two beams' reflected signals from a compact target to be easily distinguished in digital post-processing of the single receiver channel.
\end{abstract}

Index Terms-Millimeter waves, submillimeter-wavelength imaging, terahertz radar.

\section{INTRODUCTION}

$\mathbf{R}$ ADAR SYSTEMS with frequency-modulated continuous-wave operation and working at submillimeter wavelengths have shown promise for detecting concealed person-borne threats because of their centimeter-scale spatial resolution in three dimensions at standoff ranges of many meters [1]-[3]. However, shortening the image acquisition time

Manuscript received December 07, 2009; revised April 27, 2010; accepted April 28, 2010. Date of publication June 07, 2010; date of current version July 14, 2010. This work was carried out by the Jet Propulsion Laboratory (JPL), California Institute of Technology, under a contract with the National Aeronautics and Space Administration (NASA). This work was supported under a contract to the Division of Biology, California Institute of Technology, by the Naval Explosive Ordnance Disposal Technology Division, with funding provided by the Department of Defense (DoD) Physical Security Equipment Action Group (PSEAG).

N. Llombart was with the Jet Propulsion Laboratory (JPL), California Institute of Technology, Pasadena, CA 91109 USA. She is now with the Optics Department, Universidad Complutense de Madrid, Madrid 28040, Spain (e-mail: nuria.1lombart@opt.ucm.es).

K. B. Cooper, R. J. Dengler, and G. Chattopadhyay are with the Jet Propulsion Laboratory (JPL), California Institute of Technology, Pasadena, CA 91109 USA (e-mail: kbcooper@jpl.nasa.gov; no6b@no6b.jpl.nasa.gov; goutam.chattopadhyay@jpl.nasa.gov).

T. Bryllert was with the Jet Propulsion Laboratory (JPL), California Institute of Technology, Pasadena, CA 91109 USA. He is now with the Physical Electronics Laboratory, Chalmers University of Technology, Göteborg SE-412 96, Sweden (e-mail: bryllert@chalmers.se).

P. H. Siegel is with the Jet Propulsion Laboratory (JPL), California Institute of Technology, Pasadena, CA 91109 USA, and also with the Department of Biology, California Institute of Technology, Pasadena, CA 91125 USA (e-mail: phs@caltech.edu).

Color versions of one or more of the figures in this paper are available online at http://ieeexplore.ieee.org.

Digital Object Identifier 10.1109/TMTT.2010.2050106 of a terahertz radar from its current timescale of 1 min to less than $1 \mathrm{~s}$ is a major challenge. The natural path to achieving high frame rates is to fabricate a focal plane array that projects and detects multiple radar beams simultaneously, but terahertz heterodyne transceiver arrays [4], [5] have not yet been realized because of the cost and complexity of constructing many parallel submillimeter-wave sources and receivers.

Here we introduce and experimentally validate a time-delay multiplexing technique that capitalizes on the huge bandwidth and short wavelengths of a terahertz radar to simultaneously transmit and receive two spatially offset radar beams using a single heterodyne transceiver. This permits the frame rate of the imaging radar to be doubled without any additional back-end microwave or front-end terahertz components-only a handful of additional waveguide structures are needed to establish the beam trajectories. Given a sufficiently high-power terahertz source in the future, this technique can potentially be generalized to handle more than two beams, thus opening a path to achieving video-rate terahertz imaging radar without a transceiver array.

\section{System ARChITECTURE}

The electronic architecture of the terahertz imaging radar used in these experiments is based on the one described in [1], where a 30-GHz bandwidth frequency-modulated continuous-wave signal was shown to be capable of resolving the ranges of closely spaced targets with $1-\mathrm{cm}$ resolution. The only differences involve the component selection to accommodate a new operating bandwidth of $660-690 \mathrm{GHz}$ (centered on an atmospheric transmission window), and a faster microwave chirper to reach a higher pulse repetition frequency [6].

The optical architecture, however, has changed significantly to achieve time-delay beam multiplexing. Previously, the transmit and receive beams were duplexed using a 3-in-diameter silicon etalon beam splitter placed a few centimeters away from the orthogonally oriented horns of the multiplier source and mixer receiver, as shown schematically in Fig. 1(a). While effective in a single-beam imaging radar, this beam-splitter approach to duplexing is not well suited to an arrayed beam transceiver because the thin etalon is large and delicate. In addition, a second disadvantage of this approach is that the fourth port of the beam splitter was not used, and therefore, half of the terahertz energy generated by the source was lost (to space or a beam dump).

For the 660-690-GHz radar, an alternative transceiver front-end has been developed using a custom waveguide hybrid coupler as a compact substitute for the beam splitter. The coupler is built from a $2.54-\mathrm{cm}$ split-block waveguide with four 

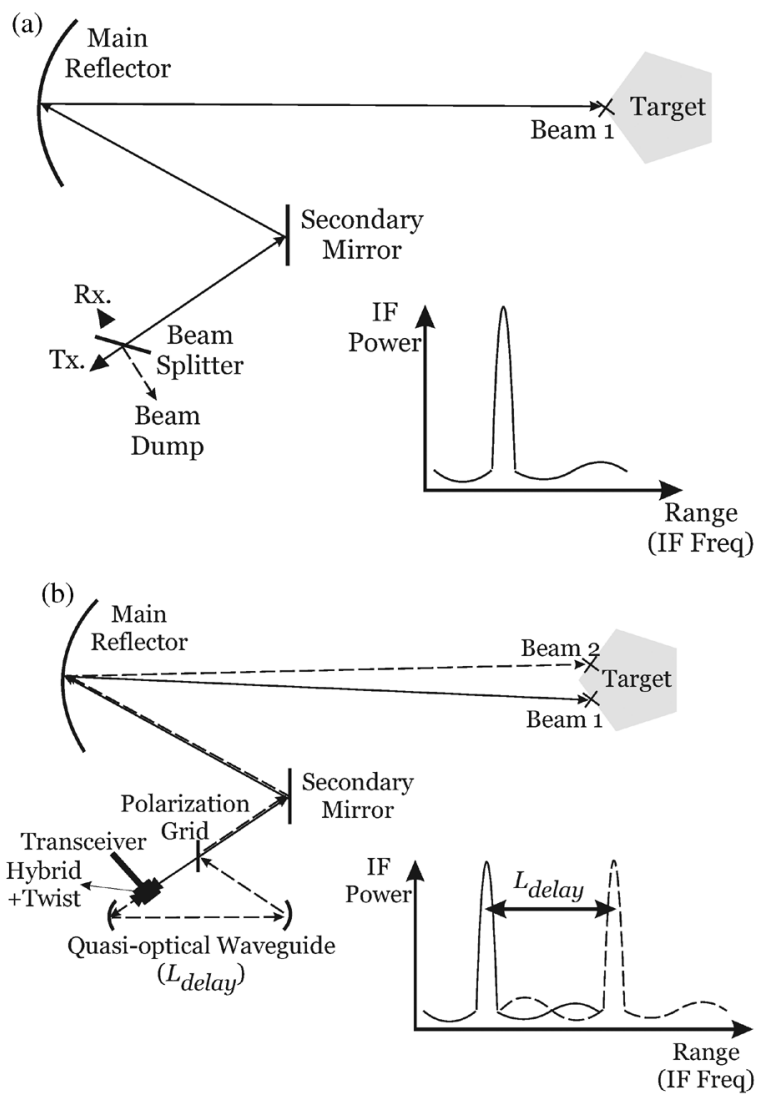

Fig. 1. System operation sketch (not to scale). (a) Terahertz imaging radar of [1]. (b) New system with the time-delay multiplexing optics.

ports consisting of two pairs with designed coupling levels of $-3 \mathrm{~dB}$ and two pairs with designed isolation of $30 \mathrm{~dB}$. The design and performance of the coupler is described in detail in Section III. The terahertz source block and receiver mixer block are connected to a pair of the coupler's isolated ports. One of the corresponding coupled ports is connected directly to a $25-\mathrm{dB}$ gain diagonal horn, and the other first connects to a short polarization twist waveguide block [7] and then a matching horn.

Aside from being smaller and more robust than a beam splitter, the hybrid coupler/twist combination is also convenient for simultaneously projecting two radar beams onto a target. This is indicated in the sketch of Fig. 1(b), where some additional optical components capture the terahertz beam previously lost in the duplexer's fourth port, and then refocus it onto the target. In this way, both of the beams from the hybrid coupler are utilized. The optical path of the first beam, represented by a solid line in Fig. 1(b), follows a path from one of the coupler's horns through a wire grid oriented to be transparent to it, then to a secondary flat mirror, and finally to the main 40-cm ellipsoidal antenna, which focuses the beam to a spot size of less than $1 \mathrm{~cm}$ at a target $4 \mathrm{~m}$ away. The second beam, on the other hand, is drawn as a dashed line in Fig. 1(b). After emerging from the second horn of the coupler/twist pair, it is first delayed by a quasi-optical waveguide of length $L_{\text {delay }}=90 \mathrm{~cm}$. Next, the second beam encounters the wire grid, but because of its orthogonal polarization with respect to the first beam, it is reflected back to the common flat secondary

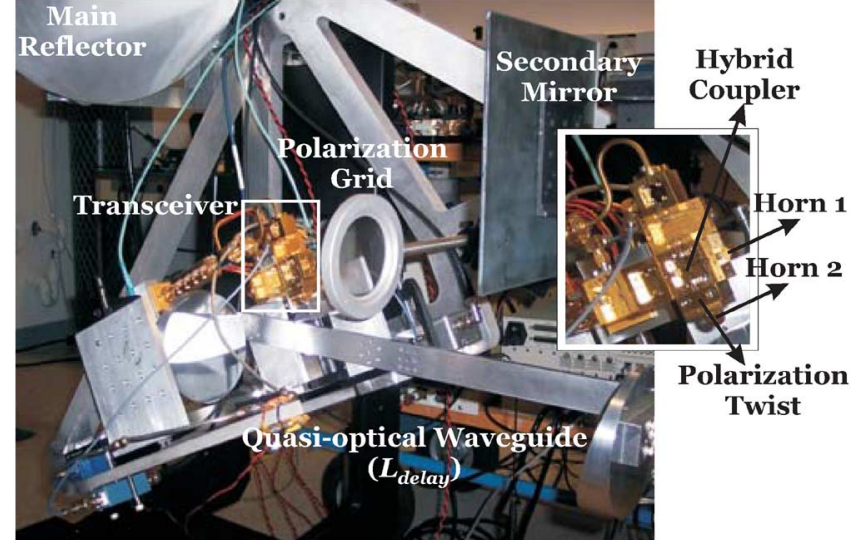

Fig. 2. Photograph of the radar system including the new components.

mirror and the ellipsoidal main mirror for focusing at a 4-m range. A photograph of these multiplexing optics is shown in Fig. 2, and futher details are provided in Section IV.

The beam-multiplexing system is designed so that the first horn is offset in the focal plane by about $0.9 \mathrm{~cm}$ from the main antenna focal point, while the effective focal point of the quasioptical waveguide matches that of the main antenna. The result is that the two beams are projected toward the target at a slightly offset angle of $0.61^{\circ}$ in elevation, corresponding to a $4.25-\mathrm{cm}$ displacement at the target range of $4 \mathrm{~m}$. Thus, while the two beams share a common source and detector, they are separated both in the 2-D cross-range space of the target focal plane and in the third range dimension by virtue of the time delay of the second beam. This means that even though both beams may be reflected by separate regions of a target at roughly the same range from the main reflector, the detected signals of the two beams will be separated by a distance $L_{\text {delay }}$ in the final IF power spectrum (in frequency-modulated continuous-wave radar, range $R$ and IF frequency $f$ are related by $R=f c / 2 K$, where $K$ is the chirp rate.) In other words, the power spectrum of the detected IF signal's power spectrum from a single target will not contain a single peaked region, as in the previous terahertz imaging radar configuration of Fig. 1(a), but instead it will generally exhibit two regions of high intensity, corresponding to the two beams, separated by a range determined by the time delay introduced in the second beam by the quasi-optical waveguide. The expected double-peaked IF spectrum is indicated in Fig. 1(b), and these peaks can then be assigned with high reliability to the appropriate beam for subsequent image reconstruction. Thus, 3-D target information can be acquired from two beams simultaneously, all without any additional terahertz sources, receivers, or analog-to-digital sampling channels.

\section{WAVEguide COMPONENTS: DESIGN AND MEASUREMENTS}

Two new waveguide components have been developed for the time-delay multiplexing 660-690-GHz imaging radar: a hybrid coupler for duplexing and a polarization twist to rotate the polarization of the second beam. The twist design was based on one already built at the Jet Propulsion Laboratory (JPL), Pasadena, CA, for operation over $220-320 \mathrm{GHz}$ [7]. For the $660-690-\mathrm{GHz}$ twist, simulations indicate that with no resistive losses, over 


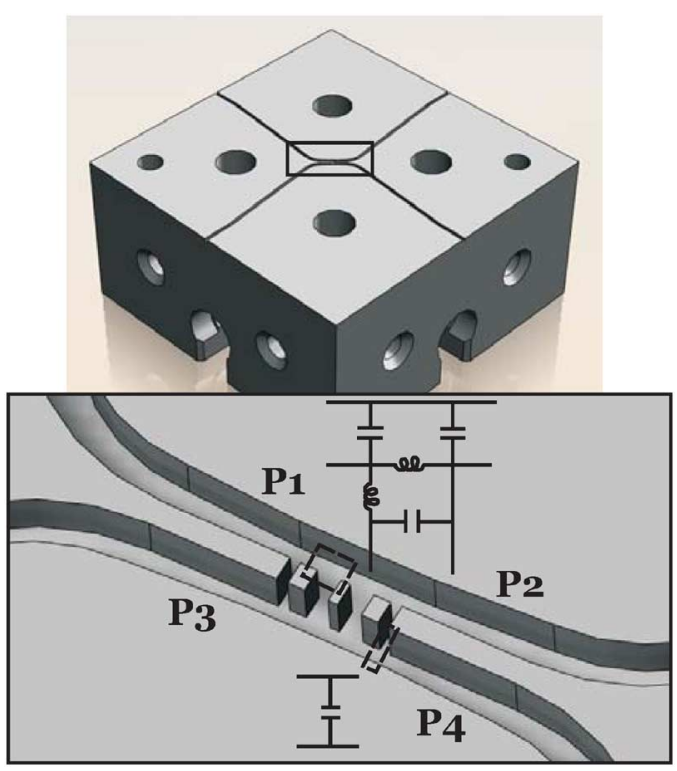

Fig. 3. Drawing of the coplanar waveguide aluminum block. The inset shows the coupler core including a couple of examples of the discontinuities and their equivalent circuits. In particular, the equivalent circuits of an $E$-plane step and T-junction are shown. The input and output waveguides have a $381-\mu \mathrm{m}$ width and $190-\mu \mathrm{m}$ height. The smallest slot dimension is $47 \mu \mathrm{m}$.

$99 \%$ of the power is coupled to the orthogonal polarization, while the rest is reflected back. However, measurements using a direct power meter reveal a significant insertion loss of around $2.4 \mathrm{~dB}$ between 660-690 GHz. Some of this may be attributed to ohmic losses from wall roughness of the gold-plated waveguide. For example, a wall roughness of $100 \mathrm{~nm}$ plus a conductivity of $10^{7} \mathrm{~S} / \mathrm{m}$ would yield an insertion loss of $1.8 \mathrm{~dB}$, according to High Frequency Structure Simulator (HFSS) simulations. In addition, some loss may come from the waveguide block's split being in the $H$-plane after the twist. Separately, vector measurements indicate a return loss below the available measurement range of around $20 \mathrm{~dB}$, which is consistent with simulations indicating a reflection of between 20-30 dB [7].

The coupler structure, shown in Fig. 3, was designed from scratch as an $E$-plane branch-line coupler because of its compatibility with a split block geometry. The bandwidth of such a coupler improves with the number of coupling branches in the waveguide interaction region, but at the cost of increasing the difficulty of fabrication. As a compromise, a four-branch design was chosen.

Optimizing a coupler design with multiple branches is very time consuming using a full-wave simulator such as HFSS, and thus an equivalent-circuit model was used to speed up the design. In this model, the hybrid core structure is divided into discontinuities represented by equivalent circuits of lumped inductors and capacitors, as described in [8], and shown in the inset of Fig. 3. For optimization, the isolation was maintained below $30 \mathrm{~dB}$ and the coupling was kept close to $-3 \mathrm{~dB}$ over the 660-690-GHz bandwidth. The design was further constrained by the tolerances of the waveguide fabrication process at these frequencies. In particular, the smallest slot dimension is limited to one-eighth of the waveguide width.

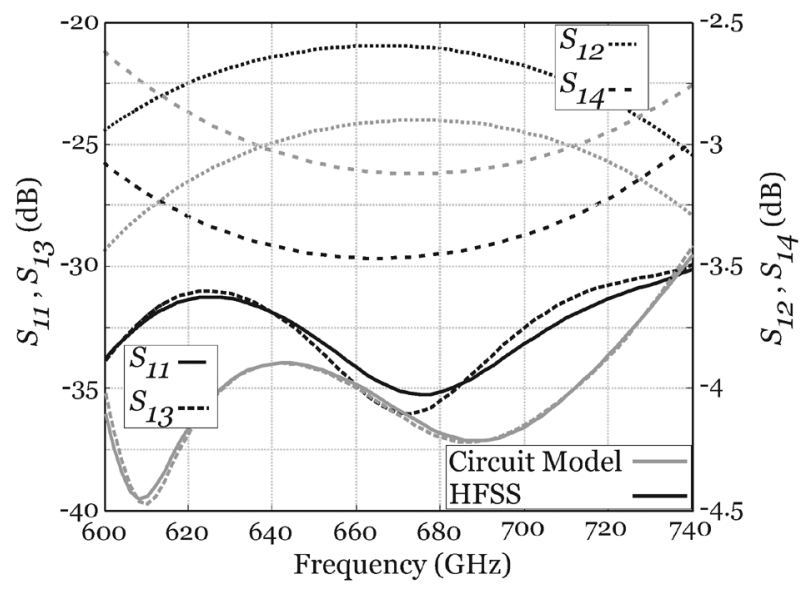

Fig. 4. Simulated $S$-parameters of the branch-line coupler.

TABLE I

MEASURED $S$-PARAMETERS OF THE BRANCH-LINE COUPLER

\begin{tabular}{|c|c||c|c|c|}
\hline Frequency & $S_{12}$ & $S_{14}$ & $\begin{array}{c}S_{13} \\
\text { (Horn) }\end{array}$ & $\begin{array}{c}S_{13} \\
\text { (Open) }\end{array}$ \\
\hline \hline $658.8 \mathrm{GHz}$ & $-6.5 \mathrm{~dB}$ & $-6.2 \mathrm{~dB}$ & $-27.9 \mathrm{~dB}$ & $-19.3 \mathrm{~dB}$ \\
\hline $662.4 \mathrm{GHz}$ & $-6.7 \mathrm{~dB}$ & $-6.6 \mathrm{~dB}$ & $-30.1 \mathrm{~dB}$ & $-24.5 \mathrm{~dB}$ \\
\hline $666.6 \mathrm{GHz}$ & $-6.6 \mathrm{~dB}$ & $-6.4 \mathrm{~dB}$ & $-25.8 \mathrm{~dB}$ & $-18.7 \mathrm{~dB}$ \\
\hline $669.6 \mathrm{GHz}$ & $-6.3 \mathrm{~dB}$ & $-6.5 \mathrm{~dB}$ & $-26.3 \mathrm{~dB}$ & $-20.9 \mathrm{~dB}$ \\
\hline $673.2 \mathrm{GHz}$ & $-7.3 \mathrm{~dB}$ & $-7.0 \mathrm{~dB}$ & $-27.2 \mathrm{~dB}$ & $-21.4 \mathrm{~dB}$ \\
\hline $676.8 \mathrm{GHz}$ & $-6.9 \mathrm{~dB}$ & $-6.8 \mathrm{~dB}$ & $-23.5 \mathrm{~dB}$ & $-18.1 \mathrm{~dB}$ \\
\hline $680.4 \mathrm{GHz}$ & $-6.1 \mathrm{~dB}$ & $-5.9 \mathrm{~dB}$ & $-24.5 \mathrm{~dB}$ & $-20.9 \mathrm{~dB}$ \\
\hline $684.0 \mathrm{GHz}$ & $-6.2 \mathrm{~dB}$ & $-6.0 \mathrm{~dB}$ & $-22.8 \mathrm{~dB}$ & $-17.9 \mathrm{~dB}$ \\
\hline $687.6 \mathrm{GHz}$ & $-6.9 \mathrm{~dB}$ & $-6.6 \mathrm{~dB}$ & $-24.6 \mathrm{~dB}$ & $-19.5 \mathrm{~dB}$ \\
\hline
\end{tabular}

This circuit model does not take into account the weak coupling of evanescent modes between adjacent coupling steps, and this may change the final performance. Therefore, once an initial design is obtained using lumped elements, a full-wave simulation of the structure with HFSS is made. Fig. 4 shows the calculated $S$-parameters of the optimized equivalent-circuit structure compared to the ones obtained using HFSS, and good agreement is achieved. More elaborate design approaches that provide very accurate results are possible [9], but this simplified design method was sufficient for the purposes of this work.

Table I shows a summary of some $S$-parameter measurements of the hybrid coupler over $660-690 \mathrm{GHz}$ using a direct power meter. Two Virginia Diodes Inc. diagonal horns were used as loads in the unused ports. The measured coupling is about 6-7 dB, which for a 3-dB coupler implies an insertion loss of around 3.5 dB. This loss is comparable with other quadrature hybrid implementations at these frequencies [10]. As with the twist, this additional loss is consistent with HFSS simulations of the waveguide wall roughness and conductivity, and it represents a significant drawback of using discrete waveguide blocks for duplexing. Integrating the source, mixer, coupler, twist, and horns into a single block to reduce the distances between components should substantially improve the losses.

Direct power measurements were not sensitive enough to accurately measure the scattered power between the isolated ports, i.e., $S_{13}$ and $S_{24}$. For example, the measured $S_{13}$ values across the operating bandwidth are shown in the third column of Table I, and they span a range from -23.5 to $-30.1 \mathrm{~dB}$. 
However, because these values are near the noise floor of the power detector, they should be considered an upper bound on the port isolation. Separately, measurements using the radar system itself for vector measurements revealed an isolation between the Tx and Rx ports of about $-28 \mathrm{~dB}$, consistent with the direct power measurements of Table I. The isolation was also confirmed to worsen when the horns from the two unconnected ports, which had served as waveguide loads, were removed, decreasing the isolation to a range from -17.9 to $-24.5 \mathrm{~dB}$, as shown in the last column of Table I. This level is comparable to that of the simulated reflection coefficient of the open waveguide minus the ohmic waveguide losses, which was $-20.5 \mathrm{~dB}$.

The performance of these waveguide components, both in terms of their loss and isolation, directly affects the noise level of the operating radar system. The impact of insertion loss is straightforward, where a combined two-way signal power reduction of $2 \times(3.5+2.4)=11.8 \mathrm{~dB}$ is caused by the hybrid coupler and the polarization twist. Also important is the effect of phase noise from the transmit signal that leaks into the receive port due to the finite isolation between the ports. While range gating the return signal from a target at a 4-m range can eliminate the signal directly coupled from the transmitter to the receiver (at effectively zero range), the phase noise of this coupled power can easily extend to IF frequencies occupied by the target signal. We find that the resulting phase noise floor is $7-10 \mathrm{~dB}$ higher than the thermal noise floor. Taken together, these effects result in a reduced signal-to-noise of about $20 \mathrm{~dB}$ that is caused by the use of a hybrid coupler and polarizing twist.

We expect to partially overcome the ohmic loss problem by designing more compact waveguide components in the future, possibly using silicon micromachining [11]. The isolation of the coupler, however, would be difficult to improve beyond the present estimated value of $-28 \mathrm{~dB}$ using any kind of waveguide design. Therefore, the signal-to-noise ratio (SNR) degradation due to phase noise would need to be addressed using a better RF circuit design, or just accepted as a moderate performance penalty. However, it is important to note that the same multiplexing technique introduced in this paper could be implemented using all quasi-optical components (beam splitter and polarization rotator), which would be practically immune to both the loss and leakage effects encountered in the all-waveguide duplexing approach, meaning that the multiplexing technique will have insignificant degradation on the SNR. We have investigated only the waveguide approach thus far because of the potential applicability to an eventual integrated array technology.

\section{QUASI-OPTICAL SYSTEM}

Depending on the actual implementation of the optical system, the beam patterns associated with the two pixels on the target can be optimized to meet different requirements. For example, it may be preferable to have the two beams separated by a certain spatial cross-distance at the target. In the current implementation, the beams are separated by $4.25 \mathrm{~cm}$ vertically and the image is formed by interleaving the two beams, reducing the complete acquisition time. Another interesting possibility is to point both pixels at the same point, but maintain orthogonal

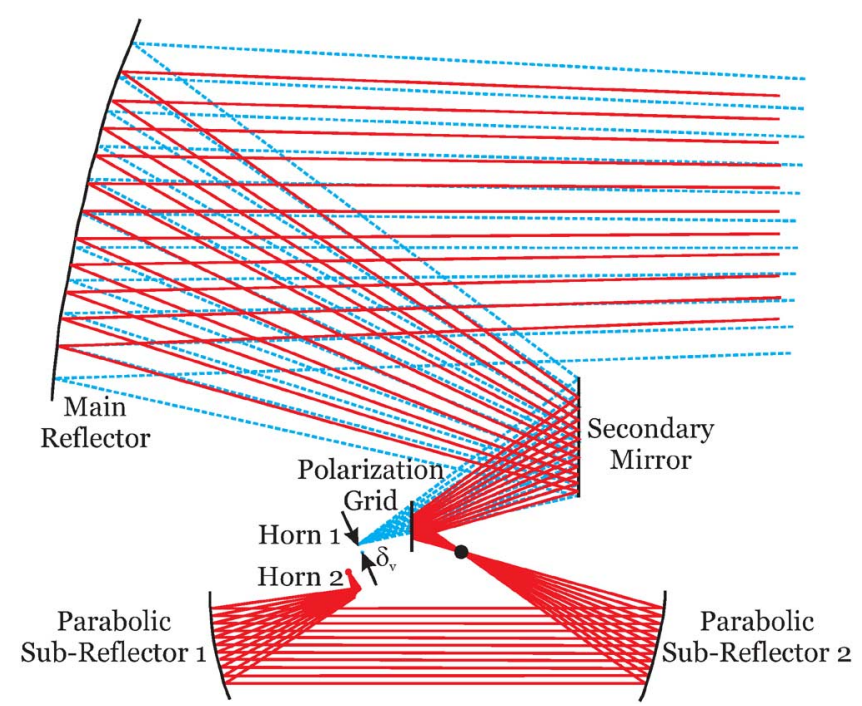

Fig. 5. Ray image of the optical system in the vertical plane. Dashed and solid lines are associated to the rays of the first and second signal, respectively.

polarizations. Polarization diversity in radar systems has long been recognized to permit a more complete inference of target parameters than is possible with a single channel radar system [12]. In this section, we describe our choice of the final optical implementation and present simulations and measurements of the optical system's performance.

\section{A. Implementation}

The main antenna system is based on the one used in [1] consisting of an offset ellipsoidal main reflector with a folded path through a secondary flat mirror. The quasi-optical waveguide refocuses the orthogonally polarized field radiated by the second horn to the focal point folded by the polarization grid; see the dot in Fig. 5. At the same time, it introduces a time delay to the second beam's waveform. One possible way of refocusing the second beam to the focal point of the main reflector is to employ a single ellipsoidal subreflector with two focuses plus a small flat mirror. However, in the current system, such a reflector would need a large offset, which would introduce significant cross polarization and rapidly affect the beam quality (i.e., half-power beamwidth and sidelobes) of off-axis feeds [13] (required in an arrayed implementation). In order to reduce the effects of a large offset, two parabolic reflectors plus a flat mirror are used for the quasi-optical waveguide, as shown in Fig. 5, where a ray tracing of both beams is provided.

The two beams need to point at slightly different offset angles in order to enable an interleaving scan approach, as discussed above. A simple way to tilt one of the beams is by displacing the first horn by $\delta_{v}=0.9 \mathrm{~cm}$ along the vertical axis of the focal plane (see Fig. 5). This off-focus distance displaces the beam of the first signal at the target $(4 \mathrm{~m})$ by approximately eight beamwidths (i.e., by $D_{v}=R_{f} \delta_{v} / F_{m} \approx 4.25 \mathrm{~cm}$, where $F_{m}$ is the distance from the focus to the center for the main reflector and $R_{f}$ is the $4 \mathrm{~m}$ focusing distance of the ellipsoid main reflector).

Using the waveguide coupler block geometry (see Fig. 3), the two beam horns are oriented $90^{\circ}$ apart on two adjacent sides of 

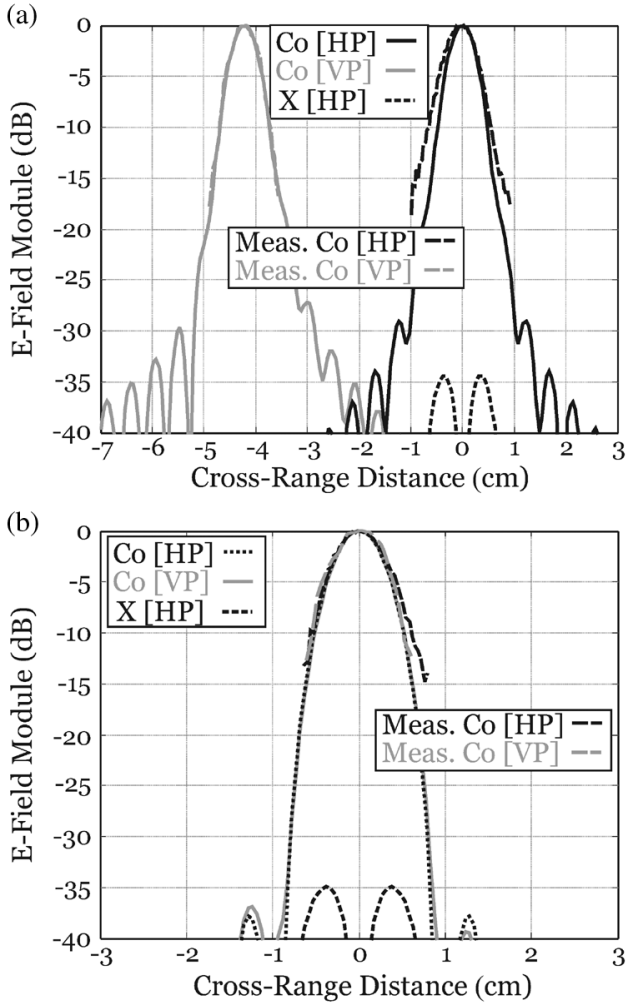

Fig. 6. Normalized beam patterns in the two main planes at $4 \mathrm{~m}(670 \mathrm{GHz})$. (a) First and (b) second beam. The HPBWs are 0.54 and $0.58 \mathrm{~cm}$, respectively. VP and HP stands for vertical and horizontal planes, respectively, while Co and $\mathrm{X}$ stand for the co-and cross-polarization components. The measured beams are also included in both (a) and (b) for comparison.

a 2.54-cm waveguide block. The waveguide twist introduces an additional spatial shift on the second horn position. This shift has been compensated with the quasi-optical waveguide by refocusing the second beam into the main aperture's focal point folded by the polarization grid. However, due to the geometrical constraints, this means that two parabolic reflectors with different f-numbers and off-axis displacements are required. This introduces some asymmetry in the illumination of the parabolic reflectors. As a consequence of this asymmetry, the main antenna is under-illuminated by the second beam, and the second pixel will have a slight increase in the half-power beamwidth, from 0.54 to $0.58 \mathrm{~cm}$. This difference can be reduced if the second horn is placed closer to the main focal point by integrating the twist and coupler in a more compact component. The different $\mathrm{f}$-numbers in the quasi-optical waveguide will also have implications in the extension of the current optical implementation to a linear array, as described at the end of this section. Below, we make the distinction between asymmetrical and symmetrical quasi-optical waveguide referring to parabolic mirrors having different or equal f-numbers, respectively.

The whole antenna system of Fig. 5 has been simulated with the commercial physical optics tool GRASP from TICRA. The beam patterns associated with the first and second beams are shown in Fig. 6. The beam patterns have been normalized to their maximums. The amplitude of the first beam is $0.37 \mathrm{~dB}$ larger than the one of the second beam, a small difference that comes from underillumination of the main aperture due to the asymmetric quasi-optical waveguide. (a)

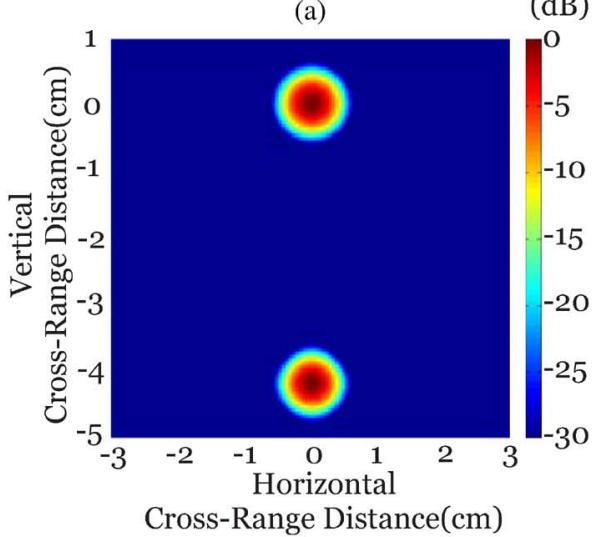

(b)

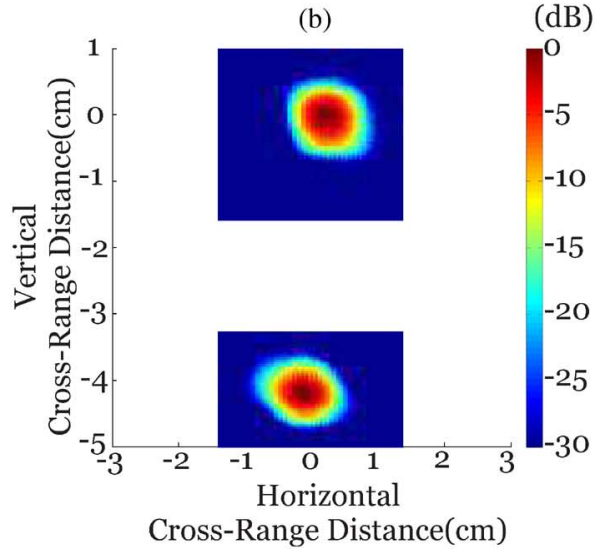

Fig. 7. $670 \mathrm{GHz}$ : (a) simulated $|E|^{4}$ beams at $4 \mathrm{~m}$. (b) Scanned radar images of the bead by the two pixels.

\section{B. Measurements}

To assess the beam profiles of the fabricated system, images were acquired of a single 3-mm-diameter gold-plated bead suspended by cotton thread at a standoff range of $4 \mathrm{~m}$. Fig. 7(a) shows the two simulated antenna radiation pattern plotted together, while Fig. 7(b) shows the two measured images of a single bead corresponding to both multiplexed signals. The received power is proportional to $|E|^{4}$ because both the transmit and received antenna patterns are the same. The measured beams in the principal planes are shown in Fig. 6 to verify the agreement with the simulations. The signal to noise level of the bead was around $35 \mathrm{~dB}$. This level is smaller than the approximately $65 \mathrm{~dB}$ measured in [1] because of the loss introduced by the waveguide components, the increase in phase noise associated with the hybrid coupler, as described above, and the shorter measurement integration time (1.7 ms compared to $12.5 \mathrm{~ms})$. The patterns shown in Fig. 7 are all normalized to their maximum value. The peak detected intensity from the second beam was approximately $7 \mathrm{~dB}$ lower than the first signal. This difference is comparable to the loss associated to the polarization twist, hybrid coupler, and optical-waveguide spill-over and gain (approximately $6 \mathrm{~dB}$ total). The additional $1 \mathrm{~dB}$ of loss may come from the use of additional mirrors, in particular an unpolished flat mirror used to redirect the second beam to the first parabolic subreflector, as well as errors in the 

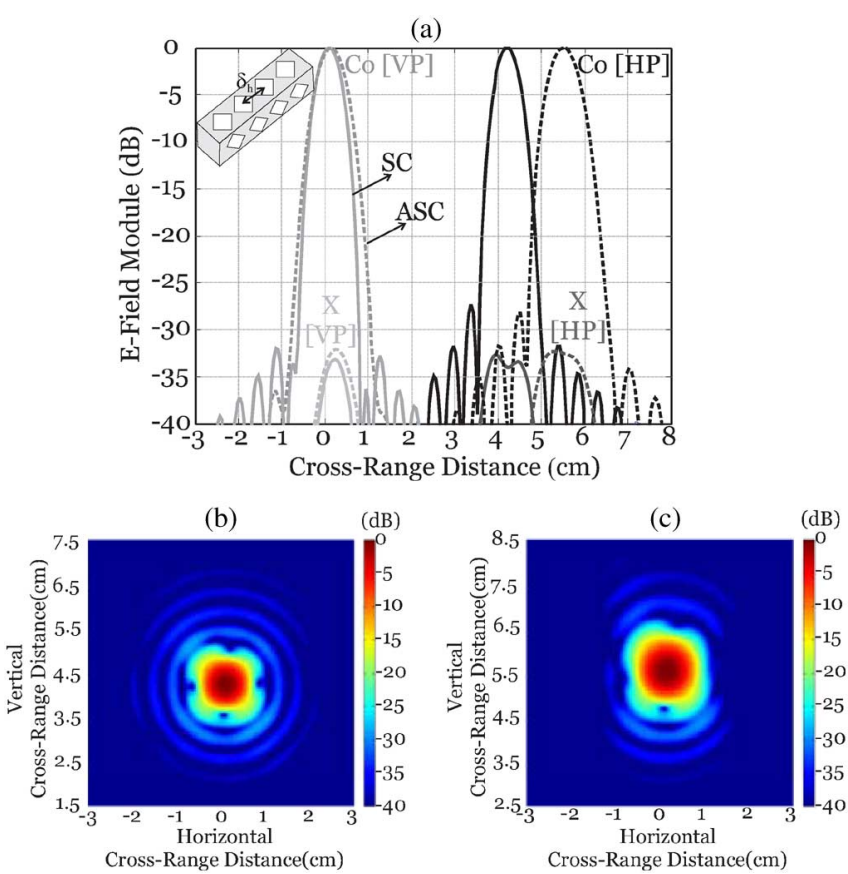

Fig. 8. Beam patterns of the multiplexed pixel at $4 \mathrm{~m}$ for an off-focal horizontal displacement of $\delta_{h}$ of $0.9 \mathrm{~cm}$. (a) Main cuts (VP and HP stands for vertical and horizontal planes, respectively, Co and X stand for the co- and cross-polarization components and SC and ASC stand for symmetric and asymmetric configurations). (b) 2-D field view of the SC case. (c) 2-D view of the ASC case. The inset in (a) shows a sketch of the linear array block consisting of two lines of horns separated by $\delta_{h}$.

optical alignment. Overall, however, the agreement between the simulated and measured beam patterns of Fig. 7 is good.

\section{Linear Array Extension}

The optical design described in Section IV-B makes use of reflectors that are offset in the vertical plane. This is convenient for a potential extension of this technique to a linear array of transceivers across the horizontal plane, and in this way, the multiplexing technique could be used to double the number of elements in the vertical direction producing two lines of pixels. In this section, we study the optical properties related to the extension of the present implementation to a linear array.

Consider first the properties associated with the first line of beams. The first array of horns will be displaced by $\delta_{v}$ from the focal line in the same way that the first beam was displaced as explained previously. Within the array, each element will be displaced a distance $\delta_{h}$ [see the inset in Fig. 8(a)]. This corresponds to a displacement of $D_{h} \approx R_{f}\left(\delta_{h} / F_{m}\right)$ at the target. However, for the second pixel, and considering the asymmetric quasi-optical waveguide, the horizontal displacement at the target range becomes $D_{h} \approx R_{f}\left(\delta_{h} / F_{m}\right)\left(F_{2} / F_{1}\right)$, where $F_{1}$ and $F_{2}$ are the focal distances of the first and second parabolic reflectors, respectively. The vertical separation, at the target, of the two lines of beams is dictated by the vertical shift $\delta_{v}$, and it is relevant to the interleaving of the lines to form the image. In contrast, the separation between two consecutive pixels within the same line is related to $\delta_{h}$ and it is relevant to the image resolution.

The actual array period is a system tradeoff between beam packing and spill-over losses, which are larger for smaller

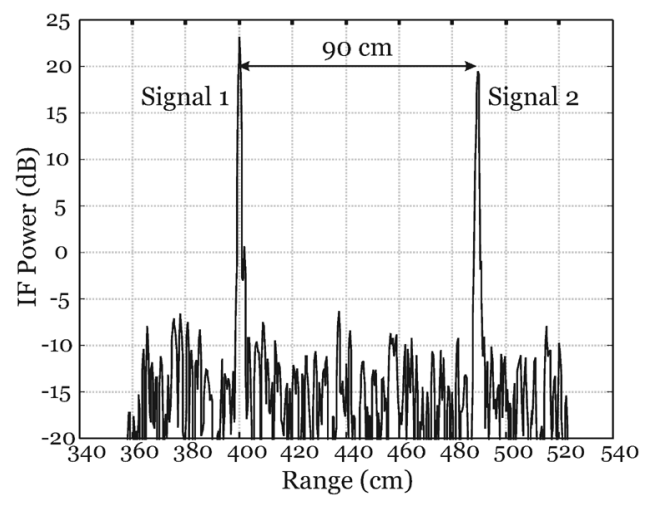

Fig. 9. Measured reflected signals from a human target placed at a standoff distance of $4 \mathrm{~m}$.

periods [14]. As an example, we consider an array period of $d \approx\left(F_{m} / D\right) \lambda_{0}$ that fully covers the line field of view. For the first line of pixels, this period translates into a on-target beam spacing of approximately $R_{f}\left(\lambda_{0} / D\right)$, whereas the second line, after propagating through the asymmetrical quasi-optical waveguide, yields a spacing of approximately $R_{f}\left(\lambda_{0} / D\right)\left(F_{2} / F_{1}\right)$. The focal distance of the second parabola is larger; therefore, the on-target beam spacing in the second line of pixels is larger with a consequence of losing resolution. As mentioned before, having more compact waveguide components will permit the design of a symmetrical quasi-optical waveguide, i.e., $F_{1}=F_{2}$, and both pixel lines will have the same beam sampling.

The design of the quasi-optical waveguide has been chosen in order to avoid the introduction of significant distortions to the off-axis beams (i.e., half-power beamwidth and sidelobe level) associated with the quasi-optical waveguide reflectors. The offaxis properties of the quasi-optical waveguide (i.e., when the feeds are displaced from the focal point) are quite good. In order to verify the beam quality, an horizontal displacement of $\delta_{h}=0.9 \mathrm{~cm}$ has been simulated with GRASP for the symmetrical and asymmetrical quasi-optical waveguides. The results are shown in Fig. 8. We can see that the beam points towards a cross-range distance of $4.25 \mathrm{~cm}$ when $F_{1}=F_{2}$ (i.e., the symmetric case), whereas it points towards $5.48 \mathrm{~cm}$ in the asymmetric case where $\left(F_{1} / F_{2}\right)=0.7762$. The fields shown in Fig. 8 exhibit low distortion because of the confocal reflector geometry between the main antenna and the second parabola of the quasi-optical waveguide [15].

\section{Time MultipleXing AND IMAGING}

The time-delay multiplexing approach has been validated experimentally. Using the terahertz front-end shown in the photograph of Fig. 2, the range-compressed spectrum detected from the dual-beam radar pointing at a person's torso at 4-m standoff range is shown in Fig. 9. As expected, two distinct signals are evident at the precise separation expected from the $90-\mathrm{cm}$ quasioptical delay line because the target surface is roughly a constant distance from the radar antenna.

To demonstrate the 3-D imaging capability of the time-delay multiplexing technique, a target scenario was set up consisting of a T-shirt clad mannequin with a mock bomb belt tied around its waist at 4-m standoff, as shown in Fig. 10(a) and (b). The 

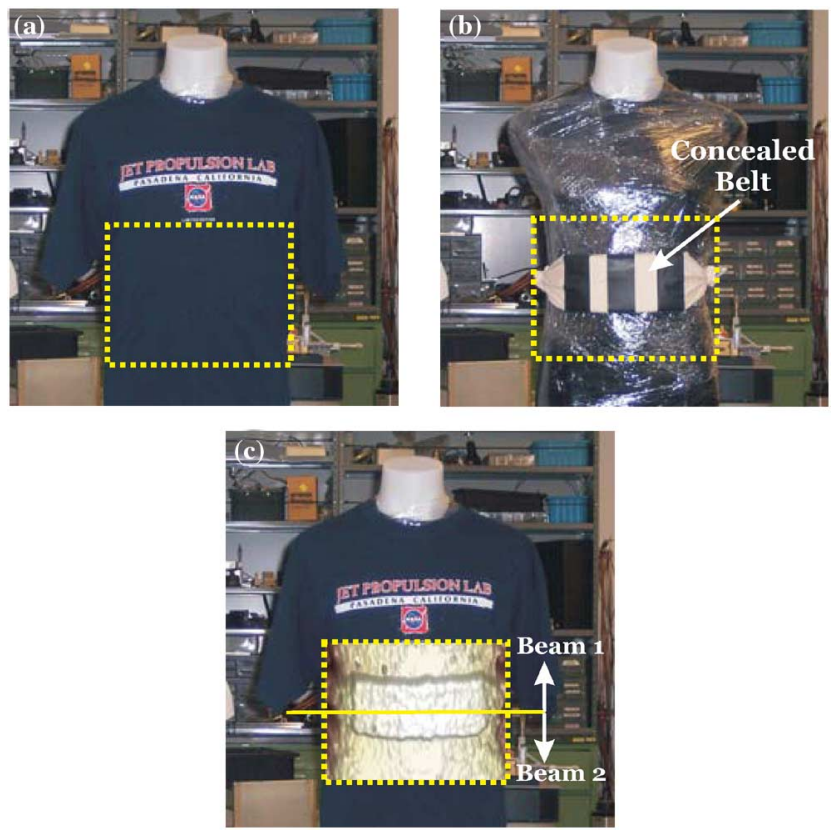

Fig. 10. Concealed belt imaging. (a) Scene. (b) Belt concealed beneath the shirt. (c) Terahertz radar image using the time-delay multiplexing technique overlaying the scene. In (a)-(c), a dashed yellow line (in online version) shows the borders of the area measured by the terahertz radar.

belt is less than 2-cm thick, and it consists of rubber sheets and packed ball bearings secured in a cotton pack wrapped in electrical tape. The plastic mannequin is wrapped in a damp T-shirt and cellophane to mimic the terahertz opacity of skin.

This scene was scanned by the two radar beams using a pulse repetition interval of $2 \mathrm{~ms}$. Calibration of the radar waveform to subtract out the effect of the radar chirp nonlinearities was done in the usual way [16] by using a highly reflective target aligned with beam 1 only. Importantly, had the two beams been generated and detected using two separate terahertz transceiver chains, then two separate calibrations would have been necessary because each chain would have introduced its own unpredictable dispersion characteristic. Here, however, only a single calibration was needed because both beams shared a common transceiver-another advantage of the time-delay multiplexing approach.

For imaging, first a simple peak association algorithm based on the $90-\mathrm{cm}$ beam delay seen in Fig. 9 was used to generate two target image pixels, separated by $4.25 \mathrm{~cm}$ vertically, for each radar pulse. After compositing the resulting data into a single contiguous image, and applying the front/back surface reconstruction algorithm described in [1], a high-resolution 3-D through-shirt image is then obtained, as shown in Fig. 10(c). This image contains $251(x) \times 96(y)=24,096$ pixels of data, which, for a single-beam implementation, would require $48 \mathrm{~s}$ to acquire at $2 \mathrm{~ms} /$ pixel. However, with two beams projected and detected per pixel, only $24 \mathrm{~s}$ of data acquisition were utilized to make the image. Overhead from mechanical scanning using the current radar platform is significant, however, typically requiring about $25 \%$ of the data acquisition time for the heavy radar stage to accelerate and decelerate at the raster scan endpoints.
With a 30-s imaging time, the utility of the present terahertz radar for real threat detection applications is still limited or nonexistent, although efforts are underway to increase the frame rate and standoff distance of the imaging radar. However, this laboratory demonstration proves that time-delay multiplexing is indeed a viable means of doubling the frame rate of an imaging radar. Automatically associating the beams with the corresponding peaks in the radar range spectrum is possible because of the relatively large $90-\mathrm{cm}$ delay built into the multiplexing optics, and regenerating a seamless 3-D through-shirt scene reconstruction works well. As higher power terahertz sources become available in the future, one can envision a similar time-delay multiplexing approach where the source power is divided among an array of beams to achieve much higher frame rates than a single-beam system.

\section{CONCLUSIONS}

The extension of a single beam to a multibeam terahertz radar imager presents a huge integration challenge at this frequency range. Here we introduced a new technique that can double the number of radar beams on a target, without any additional terahertz sources or detectors, by recovering the power of an otherwise unused beam port and introducing a relative delay between the beams. The only additional components required beyond the beam splitter (or hybrid coupler) are a polarization twist, a quasi-optical delay line, and a wire grid. Due to the terahertz radar's high operating frequency and huge bandwidth, the delay line need only to be of a modest size to permit the detected return signals from the two beams to be distinguished from one another.

Physical optics simulations were used to design the time-delay multiplexing quasi-optics, resulting in little beam-shape distortion on target. In addition, a 3-dB waveguide hybrid coupler and a waveguide polarization twist operating at 660-690 GHz were designed, fabricated, and tested. Some improvements are needed in the loss and isolation of these components, which may come from integrating them into a single block or redesigning the time-delay system using only quasi-optical components. However, the performance of the waveguide coupler and twist was more than sufficient to demonstrate that high-resolution through-shirt images of concealed threats can be made using the time-delay multiplexing technique. Future extensions of this technique might include the construction of a line array of transceivers with a single quasi-optical waveguide delay, or, if higher power sources become available, an array extension based on a single transceiver split into a multitude of delayed quasi-optical waveguides.

\section{REFERENCES}

[1] K. B. Cooper, R. J. Dengler, N. Llombart, T. Bryllert, G. Chattopadhyay, E. Schlecht, J. Gill, C. Lee, A. Skalare, I. Mehdi, and P. H. Siegel, "Penetrating 3D imaging at 4 and 25 meter range using a submillimeter-wave radar," IEEE Trans. Microw. Theory Tech., vol. 56, no. 12, pp. 2771-2778, Dec. 2008.

[2] C. Weg, W. von Spiegel, R. Henneberger, R. Zimmermann, T. Loeffler, and H. G. Roskos, "Fast active THz cameras with ranging capabilities," Int. J. Infrared Millim. Wave, vol. 30, no. 12, pp. 1281-1296, Dec. 2009. 
[3] D. M. Sheen, T. E. Hall, R. H. Severtsen, D. L. McMakin, B. K. Hatchell, and P. L. J. Valdez, "Active wideband $350 \mathrm{GHz}$ imaging system for concealed-weapon detections," Proc. SPIE-Int. Soc. Opt. Eng., vol. 7309, 2009, Art. ID 73090I.

[4] C. Groppi, C. Walker, C. Kulesa, D. Golish, J. Kloosterman, S. Weinreb, G. Jones, J. Barden, H. Mani, T. Kuiper, J. Kooi, A. Lichtenberger, T. Cecil, G. Narayanan, P. Putz, and A. Hedden, "SuperCam: A 64 pixel heterodyne array receiver for the $350 \mathrm{GHz}$ atmospheric window," in 20th Int. Space Terahertz Technol. Symp., Charlottesville, VA, Apr. 2009, pp. 90-96.

[5] G. Chattopadhyay, "Heterodyne arrays at submillimeter wavelengths," in Proc. XXVIIIth URSI Gen. Assembly, New Delhi, India, Oct. 2005.

[6] R. J. Dengler, K. B. Cooper, N. Llombart, G. Chattopadhyay, T. Bryllert, I. Mehdi, and P. H. Siegel, "Toward real-time penetrating imaging radar at $670 \mathrm{GHz}$," in IEEE MTT-S Int. Microw. Symp. Dig., Boston, MA, Jun. 2009, pp. 941-944.

[7] G. Chattopadhyay, J. S. Ward, N. Llombart, and K. B. Cooper, "Submillimeter-wave $90^{\circ}$ polarization twists for integrated waveguide circuits," IEEE Microw. Wireless Compon. Lett., submitted for publication.

[8] N. Marcuvitz, Waveguide Handbook, ser. IEE Electromagn. Wave 21. London, U.K.: IEE Press, 1986.

[9] F. Arndt, I. Ahrens, U. Papziner, U. Wiechmann, and R. Wilkeit, "Optimized E-plane T-junction series power dividers," IEEE Trans. Microw. Theory Tech., vol. MTT-35, no. 11, pp. 1052-1059, Mar. 1987.

[10] Y. Serizawa, Y. Sekimoto, M. Kamikura, W. Shan, and T. Ito, "A 400-500 GHz balanced SIS mixer with waveguide quadrature hybrid coupler," Int. J. Infrared Millim. Wave, vol. 29, no. 9, pp. 846-861, 2008.

[11] G. Chattopadhyay, J. S. Ward, H. Manohara, and R. Toda, "Deep reactive ion etching based silicon micromachined components at terahertz frequencies for space applications," in Proc. 33rd Int. Infrared, Millimeter, Terahertz Waves Conf., Pasadena, CA, Sep. 15-19, 2008, pp. $1-2$.

[12] H. A. Zebker and J. J. Van Zyl, "Imaging radar polarimetry: A review," Proc. IEEE, vol. 79, no. 11, pp. 1583-1606, Nov. 1991.

[13] Y. Rahmat-Samii, "Reflector antennas," in Antenna Handbook, Y. T. Lo and S. W. Lee, Eds. New York: Van Nostrand Reinhold, 1988.

[14] N. Llombart, A. Neto, G. Gerini, M. Bonnedal, and P. De Maagt, "Leaky wave enhanced feed arrays for the improvement of the edge of coverage gain in multibeam reflector antennas," IEEE Trans. Antennas Propag., vol. 56, no. 5, pp. 1280-1291, May 2008.

[15] N. Llombart, K. B. Cooper, R. J. Dengler, T. Bryllert, and P. H. Siegel, "Confocal ellipsoidal reflector system for mechanically scanned active terahertz imager," IEEE Trans. Antennas Propag., vol. 58, no. 6, Jun. 2010 , to be published.

[16] K. B. Cooper, R. J. Dengler, G. Chattopadhyay, E. Schlecht, J. Gill, A. Skalare, I. Mehdi, and P. H. Siegel, "A high-resolution imaging radar at $580 \mathrm{GHz}$," IEEE Microw. Wireless Compon. Lett., vol. 18, no. 1, pp. 64-66, Jan. 2008.

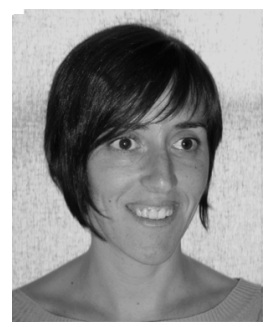

Nuria Llombart (S'06-M'07) received the Ingeniero de Telecomunicación degree and Ph.D. degree from the Universidad Politécnica de Valencia, Valencia, Spain, in 2002 and 2006, respectively.

During her Master's degree studies, she spent one year with the Friedrich-Alexander University of Erlangen-Nuremberg, Nuremberg, Germany, during which time she was also with the Fraunhofer Institute for Integrated Circuits, Erlangen, Germany. From 2002 to 2007, she was with the Antenna Unit, TNO Defence, Security and Safety Institute, The Hague, The Netherlands, initially working as a doctoral student and then as a Researcher. From 2007 to 2009, she was a Postdoctoral Fellow with the California Institute of Technology, Pasadena, where she was a member of the SWAT Group, Jet Propulsion Laboratory (JPL). Since January 2010, she has been a Ramón y Cajal Researcher with the Universidad Complutense de Madrid, Madrid, Spain. Her research interests include the analysis and design of printed array antennas, electromagnetic bandgap (EBG) structures, reflector antennas, lens antennas, and submillimeter-wave components.

Dr. Llombart was a corecipient of the 2008 H. A. Wheeler Applications Prize Paper Award of the IEEE Antennas and Propagation Society.

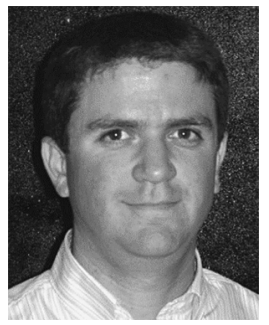

Ken B. Cooper (M'06) received the A.B. degree in physics (summa cum laude) from Harvard College, Harvard University, Cambridge, MA, in 1997, and the Ph.D. degree in physics from the California Institute of Technology, Pasadena, in 2003.

Since 2006, he has been a Member of the Technical Staff with the Jet Propulsion Laboratory (JPL), Pasadena, CA. His current research interests include submillimeter-wave radar, spectroscopy, and device physics.

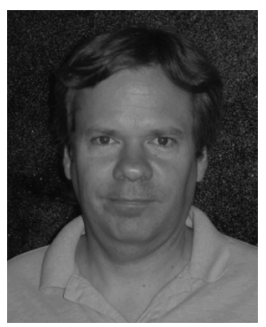

Robert J. Dengler (A'91-M'09) received the Bachelor of Science degree in electrical and computer engineering from the California State Polytechnic University, Pomona, in 1989.

In 1988, he began his work with the Jet Propulsion Laboratory (JPL), Pasadena, CA, as an Intern, where he developed beam pattern acquisition and analysis software. Since then, he has been involved in the design and construction of submillimeter-wave receivers and components, including design and fabrication of test instrumentation for submillimeter flight mixers. His recent research is focused on terahertz active imaging and heterodyne spectrometers, including design and construction of a 110-dB dynamic range biosample transmission imaging system at $2.5 \mathrm{THz}$, a high-resolution imaging radar system operating at $670 \mathrm{GHz}$, and an ultra high-sensitivity room-temperature 550-620-GHz absorption spectrometer.

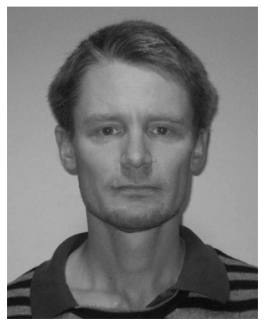

Tomas Bryllert received the M.S. degree in physics and Ph.D. degree in semiconductor physics from Lund University, Lund, Sweden, in 2000 and 2005, respectively.

In 2006, he joined the Microwave Electronics Laboratory, Chalmers University of Technology, Göteborg, Sweden, where his main research interest was device and circuit technology for terahertz frequency multipliers. From 2007 to 2009, he was with the Jet Propulsion Laboratory (JPL), Pasadena, CA, funded under a research fellowship from the Wallenberg Foundation, during which time he was involved with submillimeter-wave imaging radar and terahertz time-domain imaging systems. In September 2009, he joined the Physical Electronics Laboratory, Chalmers University of Technology, where he is involved with circuits and devices for millimeter-wave applications. Since 2007, he has also been the Chief Executive Officer (CEO) with Wasa MilliMeter Wave $\mathrm{AB}$ - a company that develops and produces millimeter-wave modules.

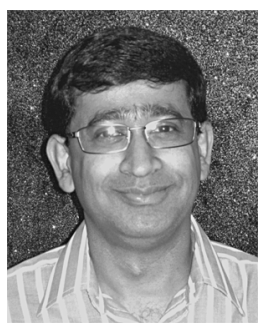

Goutam Chattopadhyay (S'93-M'99-SM'01) received the B.E. degree in electronics and telecommunication engineering from the Bengal Engineering College, Calcutta University, Calcutta, India, in 1987, the M.S. degree in electrical engineering from the University of Virginia, Charlottesville, in 1994, and the Ph.D. degree in electrical engineering from the California Institute of Technology, Pasadena, in 1999.

He is currently a Senior Member of the Technical Staff with the Jet Propulsion Laboratory (JPL), Pasadena, CA. His research interests include microwave, millimeter- and submillimeter-wave heterodyne and direct detector receivers, frequency sources and mixers in the terahertz region, antennas, SIS mixer technology, and direct detector bolometer instruments. 


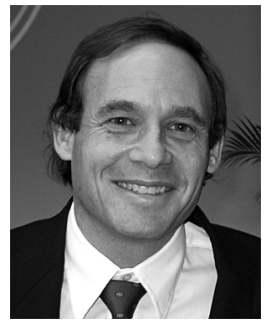

Peter H. Siegel (S'77-M'83-SM'98-F'01) received the B.A. degree in astronomy from Colgate University, Hamilton, NY, in 1976, and the M.S. degree in physics and Ph.D. degree in electrical engineering from Columbia University, New York, NY, in 1978 and 1983, respectively.

He served as a National Research Council (NRC) Fellow at the Goddard Institute for Space Studies, New York, NY, and then as a Staff Member with the Electronics Development Laboratory, National Radio Astronomy Observatory, Charlottesville, VA, until 1987. He then joined the Jet Propulsion Laboratory (JPL), Pasadena, CA, where he was involved with submillimeter-wave sensors for National Aeronautics and Space Administration (NASA) space astrophysics and Earth remote sensing applications. While with the JPL, he has been involved in four space flight missions and over 65 research and development programs. He founded, and for over 15 years, has led the large technical team Submillimeter Wave Advanced Technology (SWAT), which is focused on NASA applications of terahertz technology. In 2001, he joined the staff of the California Institute of
Technology, Pasadena, where he is a Member of Professional staff in Biology and Faculty Associate in Electrical Engineering. With the California Institute of Technology, he has expanded terahertz applications into biology and medicine, as well as into defense and security areas. He has been a Guest Editor for the Journal of Infrared, Millimeter, and Terahertz. Waves. His research interests cover all areas of terahertz technology, techniques and applications.

Dr. Siegel is an active member of the IEEE terahertz community. He has been vice-chair and chair of MTT-4, TerahertzTechnology, an IEEE Distinguished Lecturer, and continuing member of the Speaker's Bureau, as organizer and chair of seven special terahertz sessions at sequential IEEE Microwave Theory and Techniques Society (IEEE MTT-S) International Microwave Symposium (IMS) meetings, as a long-term member of the Technical Program Committee (TPC), and special guest editor for the IEEE TRANSACTIONS ON MICROWAVE THEORY AND TECHNIQUES. He is also chair of the International Organizing Committee and founding chair of the International Society of Infrared, Millimeter, and Terahertz Waves, the largest continuous forum devoted to terahertz science and technology. 